\title{
Documentarisation participative et médiation du patrimoine scientifique numérisé. Le cas des herbiers
}

Crowdsourced transcription and the mediation of scientific heritage. The case of herbaria

\section{Lisa Chupin}

\section{OpenEdition}

\section{Journals}

Édition électronique

URL : http://journals.openedition.org/edc/6499

DOl : 10.4000/edc.6499

ISSN : 2101-0366

\section{Éditeur}

Université Lille-3

\section{Édition imprimée}

Date de publication : 1 juin 2016

Pagination : $33-50$

ISBN : 978-2-917562-15-4

ISSN : $1270-6841$

\section{Référence électronique}

Lisa Chupin, « Documentarisation participative et médiation du patrimoine scientifique numérisé. Le cas des herbiers ", Études de communication [En ligne], 46 | 2016, mis en ligne le 01 juin 2018, consulté le 22 avril 2019. URL : http://journals.openedition.org/edc/6499; DOI : 10.4000/edc.6499 


\section{Documentarisation participative et médiation du patrimoine scientifique numérisé. Le cas des herbiers}

Crowdsourced transcription and the mediation of scientific heritage. The case of herbaria

\section{Lisa Chupin}

Laboratoire Dicen-IDF EA7339, Conservatoire National des Arts et Métiers lisa.chupin@cnam.fr 
Nous étudions les enjeux de la documentarisation participative d'herbiers numérisés pour la médiation du patrimoine scientifique. Il s'agit de questionner l'opérativité sociale et symbolique exercée par un dispositif régi par l'objectif de co-construction d'une médiation documentaire adéquate aux objectifs scientifiques de l'exploitation des collections. Nous montrons qu'au-delà de son efficacité documentaire, la plateforme donne lieu à une forme socialisée de médiation des savoirs concernant les collections, et que la participation à la documentarisation des images suscite l'intérêt pour les spécimens en facilitant leur mise en relation avec les territoires et les acteurs à l'origine de leur production.

Mots-clés : médiation documentaire, médiation des savoirs, crowdsourcing, étiquette, herbier.
We analyse the extent to which an online platform for the collaborative transcription of digitised herbaria can improve the mediation of scientific heritage. We study the social and symbolic operativity of this system, whose purpose is to co-construct a documentary mediation that would fit the scientific goals of exploiting the collections. We show that beyond its documentary efficiency, the platform gives rise to a socialised form of knowledge mediation. Moreover, we show that participation in the documentarisation of images increases interest in the collections by facilitating the connection between a specimen and the territories and actors responsible for its production.

Keywords: documentary mediation, knowledge mediation, crowdsourcing, label, herbarium. 
La numérisation du patrimoine brouille les frontières entre les dispositifs documentaires utilisés dans une démarche de conservation et ceux qui sont destinés à intéresser un public avec une intention de médiation (Després-Lonnet, 2014). Il existe un risque de minimiser le travail de communication nécessaire à favoriser l'appropriation par différents publics de documents devenus accessibles à tous sur internet mais destinés initialement à des publics et usages spécifiques: on le voit dans l'analyse proposée par Marie Després-Lonnet (2000) de la conception d'une interface de consultation visant à permettre l'appropriation par un public non spécialiste de fonds d'images réunis par des historiens, qui constitue selon elle un projet de «vulgarisation scientifique». Surmonter ce risque suppose d'envisager de nouvelles formes de médiation du patrimoine, autour de la recherche de convergences et de complémentarités entre dispositifs et actions de médiation issues de différentes traditions professionnelles. C'est sous cet angle que nous abordons les plateformes qui proposent aux internautes non seulement de consulter des collections exploitées essentiellement dans un cadre scientifique avant leur numérisation, mais aussi de s'approprier des pratiques entrant dans le traitement documentaire de ces collections. Pour désigner les formes de participation proposées aux internautes dans toute leur diversité (indexation, traduction, transcription, correction...), nous utilisons le terme de «documentarisation» qui renvoie à l'ensemble des opérations nécessaires au traitement documentaire, matériel et intellectuel, visant à «optimiser l'usage du document en permettant un meilleur accès à son contenu et une meilleure mise en contexte» (Salaün, 2007). À partir de l'exemple de la transcription des étiquettes d'herbiers visant à enrichir une base de données d'images issues de leur numérisation, nous cherchons à montrer comment l'apprentissage des normes et techniques de documentation suscite l'intérêt pour les collections scientifiques chez des internautes non spécialistes. Nous questionnons les formes d'interprétation des documents placés dans ce contexte spécifique de documentarisation "participative» (Casemajor Loustau, 2011). Nous étudions les reconfigurations des médiations documentaire, culturelle et scientifique à l'œuvre dans ce dispositif et questionnons leur mobilisation complémentaire comme une clé de son efficacité.

Le présent travail a été rendu possible grâce à un partenariat entre un laboratoire de sciences de l'information et de la communication et différentes institutions conservant des collections d'histoire naturelle, réunis au sein de I'Infrastructure Nationale de Biologie et Santé e-ReColNat (ANR-11-INBS-0004) constituée en vue de la numérisation des collections. Le partenariat a pour objectif le développement d'un site web permettant l'exploitation des spécimens en ligne et la mobilisation des internautes dans le traitement documentaire des images issues de la numérisation. La présente analyse ne porte pas sur les dimensions spécifiques au travail de conception du site web: elle se nourrit d'une étude préalable des usages générés par la première version de la plateforme de transcription d'herbiers de l'infrastructure e-ReColNat (2013), "Les Herbonautes» (MNHN, 2012). Nous commençons par présenter en quoi 
le fait de proposer aux internautes le traitement des collections scientifiques numérisées affecte différentes traditions de médiation, avant d'exposer la méthodologie qui en découle pour l'analyse du dispositif "Les Herbonautes"; nous présentons enfin les spécificités des médiations qui conditionnent l'efficacité documentaire du dispositif.

1.

\section{La documentarisation participative à la croisée de différentes formes de médiation du patrimoine}

\subsection{Les convergences entre médiations documentaire et culturelle}

Différents travaux cherchant à penser de nouvelles complémentarités entre documentation et médiation culturelle commencent par rappeler ce qui les distingue. Selon Marie Després-Lonnet (2014), la documentation est réalisée en vue "d'exigences d'inventaire et de connaissance érudite des collections", souvent internes à l'institution, et se distingue des actions de médiation destinées à faire découvrir un patrimoine à des publics caractérisés par leur diversité. Isabelle Fabre (2012) rappelle la spécificité du point de vue adopté pour décrire les objets des collections par les documentalistes, qui se démarque, par son effort d'objectivité et de normalisation, des points de vue disciplinaires. Ces distinctions sont un préalable pour souligner la complémentarité entre documentation et médiation culturelle, dont peut témoigner par exemple le rôle des étiquettes et de différents écrits d'exposition réalisés par des acteurs de la médiation culturelle à partir de la documentation. Selon Julie Desjardins et Daniel Jacobi (1992), l'étiquette est constitutive de la valeur d'un objet inséré dans une collection: "avec la disparition de son étiquette, l'unité de la collection est privée tout à la fois, de sa mémoire, de sa place dans l'inventaire et en définitive c'est sa signification qui devient incertaine»; mais on retrouve aussi l'étiquette, après des réécritures plus ou moins importantes, comme un élément essentiel dans le dispositif de l'exposition, assurant une «médiation entre l'objet et le visiteur » et contribuant «à l'élaboration du sens ».

Cette complémentarité est d'autant plus manifeste si, comme Vincent Liquète, Cécile Gardiès et Isabelle Fabre, on envisage les productions issues des traitements documentaires comme différents objets constitutifs d'un processus qui «dépasse la simple transmission de l'information pour aller vers une construction de liens entre besoin et usage d'information, et permette ainsi à l'individu de transformer l'information en connaissance " (Liquète et al., 2010) - processus de «médiation documentaire» qui constitue alors aussi une "médiation des savoirs». Les dispositifs de médiation documentaire peuvent viser à surmonter la distance entre langage naturel et langage de description des professionnels, de même que la médiation culturelle, selon Jean Davallon 
(2004), tend à combler l'écart entre «deux univers étrangers l'un à l'autre (celui du public et celui, disons, de l'objet culturel) dans le but précisément de permettre une appropriation du second par le premier "; l'objectif de faire "accéder un public à [des savoirs] » est commun aux deux démarches. Isabelle Fabre revient sur ces distinctions pour décrire les pratiques des documentalistes d'un musée d'art, qui complètent le travail réalisé pour les acteurs de la médiation culturelle dans un blog où la médiation documentaire se prolonge, plus librement, par des «médiations cognitives, médiations actives » et «créatives». Cette recherche de conceptions englobant les catégories de médiation culturelle et documentaire est utile pour appréhender les enjeux de l'ouverture aux internautes de la documentarisation des collections numérisées pour en faciliter l'appropriation: il s'agit d'envisager comment sont reconfigurées les médiations documentaire et culturelle, quand la documentarisation vise à être accessible à tous et au service de la compréhension des documents.

1.2. De l'efficacité documentaire à l'opérativité sociale et symbolique de la documentarisation participative

L'ouverture des catalogues des collections à l'indexation sociale comme complément de l'indexation professionnelle peut être perçue comme une transformation du dispositif de médiation documentaire, visant à mieux surmonter les différences de représentation entre usagers et professionnels réalisant l'indexation: les expérimentations réalisées en ce sens dans des musées d'art partenaires du projet Steve Museum attestent d'améliorations effectives de la recherche documentaire grâce à la participation des internautes (Trant, 2008). Mais on peut considérer sous un autre angle ces mêmes expériences, comme le fait Corinne Welger-Barboza (2013), qui souligne qu'elles ont pour effet de rendre visible dans un catalogue le "regard effectif des publics sur les œuvres» (p.203), laissant envisager les enjeux de l'indexation sociale pour la production de nouvelles interprétations. La participation des internautes à la documentarisation de fonds numérisés passe d'un enjeu d'efficacité des recherches documentaires à une perspective culturelle si on prend la mesure de ses effets sur la mobilisation de nouveaux publics: Mia Ridge (2013) signale l'intérêt que présentent, pour tisser des liens forts entre l'institution et ses usagers, des dispositifs qui proposent des tâches évolutives, orientées à la réalisation de projets proposés par l'institution détentrice des collections ou en association avec une équipe de recherche en vue de leur exploitation. Toutefois, si de nombreux dispositifs proposent des opérations de documentarisation ouvertes à tout internaute sans condition de compétence, s'inspirant des plateformes de "crowdsourcing » adaptées aux objectifs des institutions, ce sont des communautés de contributeurs proches des usagers réguliers des institutions qui se mobilisent (Owens, 2014).

Considérer les sites ouvrant la documentarisation des collections numérisées aux internautes comme des dispositifs de médiation du patrimoine conduit à interroger de concert leur opérativité sociale et symbolique, en ques- 
tionnant les formes d'interprétation faites des documents par ces nouveaux collectifs en fonction des dispositifs et des tâches proposées. C'est l'approche que propose Nathalie Casemajor Loustau (2011), et avec laquelle elle analyse les nouveaux rapports qui s'instaurent entre contributeurs et images des fonds photographiques de Bibliothèques Archives Canada, en décrivant les figures d'usagers "témoins » et d'usagers «experts » qui apportent des contributions respectivement sur la base de leur expérience vécue ou de leurs connaissances, notamment historiques. Ces enjeux des dispositifs participatifs de médiation du patrimoine, qui conduisent à envisager des modèles de production de savoir tirant parti de la contribution des internautes (Dunn et Hedges, 2014, p. 236), invitent à questionner la convergence qu'ils rendent possible entre médiations documentaire et culturelle, et médiation des savoirs - en particulier médiation scientifique dans le cas des collections d'histoire naturelle numérisées.

1.3. Documentarisation participative et nouvelles formes de médiation scientifique

Si l'on cherche à situer les modalités de production et d'acquisition de savoirs à l'œuvre dans les projets de documentarisation participative, on peut constater une situation de communication inversée par rapport aux formes de médiation des savoirs scientifiques qui portent traditionnellement sur des connaissances déjà produites et validées par les chercheurs (Jacobi, 2007), qu'il s'agisse de les transmettre ou d'en faire discuter les enjeux pour la société. Participer à des tâches documentaires conduit les internautes à produire une partie des données qui constitueront le catalogue des collections, alors que c'est notamment sur lui que s'appuient les concepteurs d'exposition. La prise en charge de tâches documentaires par les publics se rapproche en revanche de la tradition des pratiques scientifiques amateurs ayant joué un rôle important dans la constitution des collections, et trouvant de nouvelles opportunités de se développer grâce aux outils numériques (Wiggins et Crowston, 2011). Toutefois, si l'on prend en compte les modalités de régulation de la participation en vigueur dans les plateformes, souvent hiérarchiques et d'autant plus strictes qu'elles constituent une nouvelle forme de protocole commun nécessaire à cadrer la participation pour assurer une production de données scientifiquement exploitables, le renversement dans la position du contributeur en situation de produire des données est à nuancer. Les formes d'encadrement de la participation utiles à la production de données conformes aux normes institutionnelles et exploitables pourraient concourir à reconstituer dans les plateformes participatives les caractéristiques d'une médiation scientifique fondée sur la transmission d'un savoir validé par l'institution à ses publics.

Ces différents enjeux de la documentarisation participative nous conduisent à étudier le phénomène sous l'angle de la convergence et de la reconfiguration de différentes traditions professionnelles de médiation, scientifique, culturelle et documentaire. À partir du cas des herbiers, nous cherchons à expliquer comment la plateforme organise différentes médiations nécessaires 
à son fonctionnement. Nous faisons l'hypothèse selon laquelle l'«efficacité documentaire» du dispositif (Casemajor Loustau, 2011) dépend de sa capacité à mobiliser des publics non spécialistes autour d'un objectif documentaire au service du patrimoine, et à permettre d'acquérir les connaissances nécessaires à la participation.

\section{2. \\ Étudier les médiations à l'œuvre dans un dispositif de documentarisation participative}

\subsection{Les spécificités documentaires des herbiers}

Un préalable, pour comprendre la documentarisation participative d'herbiers comme médiation des savoirs, est de prendre en compte les caractéristiques de ces documents, qui expliquent en partie l'intérêt du travail sur leurs étiquettes. Les herbiers associent aux spécimens l'information collectée sur le terrain et le résultat des expertises dont ils ont fait l'objet: ces informations sont consignées dans des étiquettes, associées à chaque spécimen et essentielles à son exploitation scientifique. Les planches d'herbiers des institutions qui les conservent proviennent de multiples collections particulières (Pérez et Pignal, 2013), et portent la marque - et parfois la signature- des acteurs ayant participé à leur constitution. Documentariser les images issues de la numérisation des planches consiste à transcrire les informations des étiquettes dans une base de données, ce qui suppose, préalablement à la saisie, de les déchiffrer (Heaton et Proulx, 2012). Confier une partie de ce travail à des internautes demande de les initier à la lecture du document scientifique et aux recherches d'information qu'elle nécessite.

\subsection{Sélection du corpus et recueil des données}

Pour examiner les médiations à l'œuvre dans la documentarisation participative d'herbiers, nous avons d'abord analysé dans différentes plateformes les stratégies élaborées par les institutions pour répondre à leur objectif d'informatisation d'herbiers (Zacklad et Chupin, 2015). Nous avons étudié le discours adressé aux internautes, les formes de participation (en observant les tâches et les outils de communication proposés), de rétribution symbolique, et de validation des contributions. Appréhender les médiations effectivement à l'œuvre dans ces dispositifs demande d'étudier leur appropriation par les animateurs et les contributeurs. Nous avons étudié les formes d'interaction et d'action collective que ces différentes plateformes suscitent, en nous fondant sur une conception de la mobilisation de collectifs médiatisés par des artefacts documentaires (Zacklad, 2015). Pour comprendre l'interprétation des documents proposés et l'acquisition de connaissances dans ces collectifs, 
nous avons restreint notre étude à la plateforme des «Herbonautes» (MNHN, 2012), la seule pour laquelle nous pouvions disposer de données suffisantes sur les contributeurs. Nous avons réalisé une dizaine d'entretiens semi-directifs auprès de contributeurs et d'animateurs, centrés sur les techniques mobilisées pour déchiffrer les documents et les motivations qui sous-tendent la participation. Nous avons aussi réalisé un questionnaire en ligne: parmi les 150 réponses collectées entre fin mai 2014 et début janvier 2015, nous avons utilisé exclusivement celles des 85 répondants ayant contribué au site. Enfin, nous avons analysé les messages échangés entre contributeurs sur les espaces de discussion du site qui renseignent sur l'activité de transcription et les ressorts sociaux qu'elle mobilise.

\subsection{Analyse du corpus}

Nous analysons les différents textes de la plateforme, vecteurs des connaissances et des échanges d'informations nécessaires à l'activité proposée. Hormis les pages fixes éditées par les responsables du site, présentant les objectifs et les consignes générales (accessibles sur toutes les pages depuis un bandeau supérieur), les textes rédigés par les animateurs sont produits à mesure des nouvelles tâches proposées. Les animateurs disposent d'un espace de présentation des «missions» dont ils ont la charge (onglet «présentation» dans la figure1). Chaque mission consiste en une sélection de spécimens à transcrire partageant une unité thématique, par exemple "Plantes d'Angola», "Plantes toxiques». À chaque mission sont associés un forum de discussion, ainsi que différents outils statistiques permettant de suivre l'avancée de la mission (voir figure1).

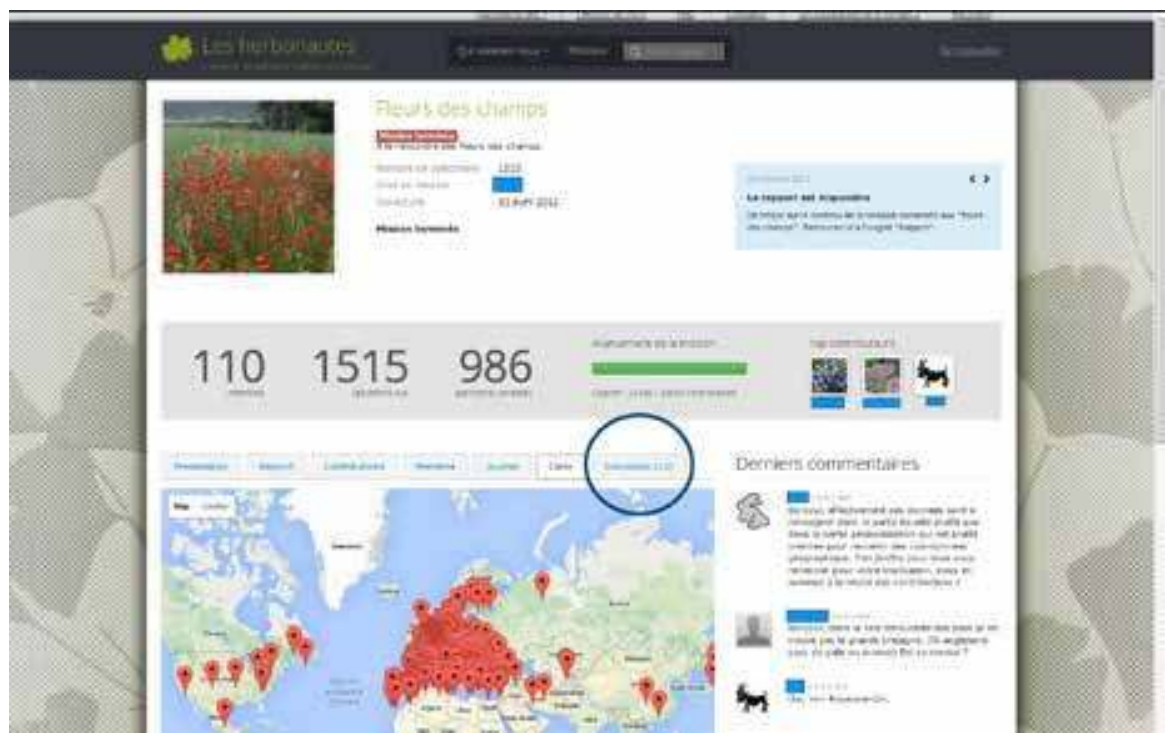

Figure 1: page de la mission «Fleurs des champs» (capture d'écran) 
Dans la moitié supérieure de la page sont indiqués le nombre de contributeurs et les résultats de la mission (nombre de spécimens transcrits). La partie inférieure de l'écran montre la carte de répartition des spécimens géolocalisés ainsi que, sur la droite, les derniers messages. L'onglet discussion (entouré) donne accès au forum de la mission.

Chaque page consacrée à une planche à transcrire (voir figure2) est dotée d'un forum, qui, lui, n'est visible que des contributeurs ayant participé à la transcription de la planche.

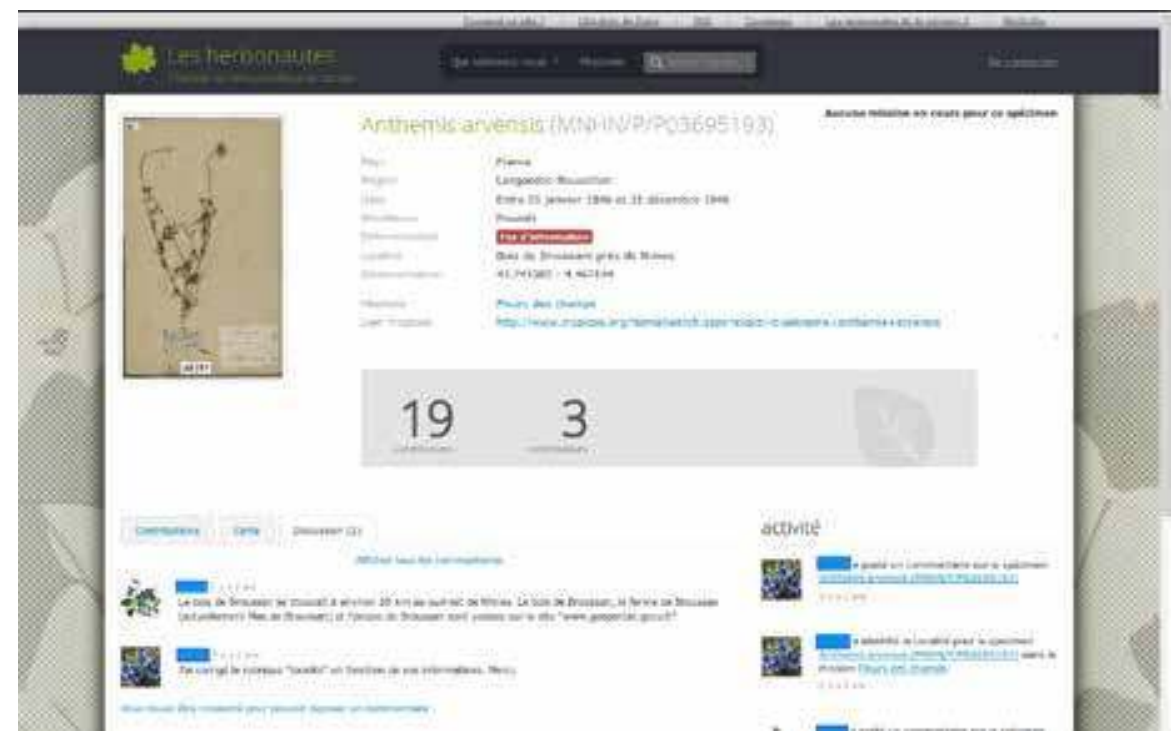

Figure 2: page du spécimen nP02115067 (capture d'écran)

La moitié supérieure de l'écran récapitule les valeurs validées pour chaque champ, et indique le total des 19 contributions (une contribution = un champ de saisie complété) réalisées par 3 contributeurs sur ce spécimen. La partie inférieure de l'écran montre l'espace de discussion consacré à cette planche, avec un échange entre deux herbonautes (pseudos effacés) permettant de déterminer la localité correcte de la collecte.

Étant donné la taille du corpus des messages sur les différents forums du site (15 000 commentaires environ sur les 20 mois de fonctionnement étudiés, entre novembre 2012 et juin 2014), nous avons cherché à produire une vue synthétique des thématiques dans les différents espaces d'échanges. Nous avons réalisé une analyse lexicale dénombrant les principales formes du corpus après lemmatisation, avec le logiciel Iramuteq (Ratinaud, 2005), dont nous présenterons les résultats sous forme d'un nuage des formes les plus fréquentes de chaque corpus. Nous avons par ailleurs lu et annoté manuellement 
les contenus produits par les animateurs dans les espaces de présentation de 5 missions, ainsi que l'ensemble des messages dans deux forums de mission, ce qui nous a permis de distinguer des caractéristiques propres à chacun de ces espaces en termes de contenus thématiques et d'acteurs qui y interviennent.

\section{3. \\ Du défi documentaire à la découverte des collections: des médiations reconfigurées}

\subsection{Une participation cadrée par les normes documentaires institutionnelles}

La documentarisation participative des herbiers associe renouvellement et reproduction des formes de communication qui interviennent dans la médiation scientifique. L'appel à la participation n'efface pas la dissymétrie entre ceux qui le formulent et les internautes qui y répondent, et conduit à développer des moyens de diffusion des connaissances documentaires botaniques qui partagent les codes de la vulgarisation scientifique, par exemple sous la forme ludique de quiz pédagogiques (voir Chupin, 2014). Tout en constituant un espace régi par des modalités de communication et de régulation spécifiques, la plateforme reste imprégnée par les normes institutionnelles qui régissent la lecture des documents proposés, mais aussi l'ordre de présentation des documents aux internautes, qui porte la trace du classement des collections selon des critères scientifiques, caractéristiques de la "communication institutionnelle» (Davallon, 1998). Certes, on peut voir les «missions" comme une façon de déroger à cet ordre de présentation des collections, puisqu'elles offrent une sélection d'images associées à une thématique susceptible d'intéresser les contributeurs. Toutefois, cette fantaisie a des limites: la sélection des spécimens de la mission se fait en fonction d'un besoin scientifique jugé prioritaire par l'un des animateurs de la plateforme. De plus, la thématique commune aux spécimens d'une mission est une réécriture, sous forme attrayante, d'une requête dans la base de données existante. La sélection des images constituant une mission se fait à partir du catalogue (constitué d'une indexation sommaire réalisée au moment de la numérisation). Comme dans le cas des expositions virtuelles constituées à partir de notices issues du catalogue de la base Joconde, analysé par Marie Després-Lonnet (2009), les recherches d'une présentation innovante des images trouvent ici des limites dans la nécessité de recourir aux outils documentaires existants pour constituer la sélection. En outre, la présence de l'institution passe par le cadrage de l'interprétation des documents à transcrire, que l'on trouve non seulement dans les consignes visant à respecter les normes des bases de données botaniques, mais aussi dans l'interface de transcription: la saisie est limitée aux champs proposés - alors que la planche d'herbier peut contenir les multiples notes prises par les récolteurs - avec pour certains champs des référentiels terminologiques limitant encore les possibilités de saisie et les erreurs potentielles. 
Malgré ces cadrages multiformes, l'appropriation des savoirs documentaires spécialisés essentiels à la lecture du document ne serait pas efficace sans ses relais communautaires. L'importance de l'accompagnement dans l'apprentissage de la lecture de l'étiquette et la compréhension du dispositif de transcription ressort de la "désorientation» (Souchier et al., 2003) observée chez les personnes découvrant le site. Elle transparaît également dans certains commentaires et dans les témoignages des contributeurs les plus investis revenant sur leurs premières expériences $d^{\prime}$ «herbonaute».

\subsection{Une médiation des savoirs documentaires botaniques communautaire}

Les interactions entre contributeurs et avec les animateurs scientifiques sont centrales pour les apprentissages nécessaires à la transcription. Les contributeurs les plus réguliers sont aussi ceux qui s'investissent le plus dans les échanges (Zacklad et Chupin, 2015), participant à définir et à rappeler aux novices les règles de transcription les plus efficaces pour la communauté-qui peuvent se différencier des conventions adoptées par les professionnels. Le forum associé à chaque mission donne lieu à des échanges de questions et réponses entre contributeurs et avec l'animateur dit «chef de mission ", centrés sur l'application des consignes selon les multiples cas qui se présentent et les singularités des documents, ainsi qu'au signalement d'anomalies constatées dans le fonctionnement du site. Le forum sert à mutualiser les difficultés rencontrées, prises en charge par l'animateur qui répond à la plupart des questions, avec toutefois aussi des initiatives de certains contributeurs réguliers venant directement en aide à d'autres. Dans cet espace, l'animateur (qui peut être un chercheur, un conservateur d'herbier, ou encore un membre de Tela Botanica, "réseau des botanistes francophones » partenaire de l'infrastructure e-ReColNat et investi dans le recensement des herbiers de France et l'animation de la plateforme) accompagne la pratique des contributeurs sur la base de son expérience des collections, en explicitant par exemple pour certaines règles le travail de documentation du musée qui justifie les consignes de transcription. La diversité des animateurs renforce le caractère communautaire de la médiation scientifique à l'opposé d'une transmission d'un savoir institutionnel univoque.

La transcription de l'étiquette d'un même spécimen donne lieu à des discussions entre les contributeurs s'étant vu proposer la même planche, dans le forum qui lui est associé. Alors que l'on s'adresse à l'animateur dans le forum de la mission (d'où la position centrale de la salutation dans la figure 3-a), les messages associés aux spécimens sont destinés seulement aux autres contributeurs. Le nombre de messages laissés dans les forums associés à des spécimens (13000environ), excède largement celui des commentaires (autour de 1500) dans les espaces de discussion des missions visibles de tous: les interactions productrices de connaissances par la mutualisation des ressources et l'argumentation des choix de transcription se font donc largement hors du contrôle des animateurs. 
L'étude de ces messages montre la constitution d'un collectif dont les membres sont mis en relation par ces échanges; elle permet aussi d'avoir une idée des questionnements suscités par l'examen des spécimens et donc des opérations d'interprétation auxquelles donne lieu la documentarisation. Les messages associés aux spécimens permettent aux contributeurs d'expliquer leurs choix de transcription, de signaler un doute, de formuler une hypothèse, pour «trouver» le «nom» du botaniste, du récolteur ou d'une localité inconnue ou illisible (voir figure 3-b). Leur activité est centrée sur la compréhension de I'«étiquette» au centre de l'attention de l'internaute - et du nuage de formes (figure 3-b), d'autant que le terme de "spécimen » est employé aussi dans les échanges pour désigner la planche et non l'échantillon. Le déchiffrement des informations de l'étiquette peut requérir la consultation d'autres sources d'information comme les bases de notices de botanistes, ou la consultation du catalogue du Muséum - dont les URL sont fréquemment citées dans les commentaires (forme «mnhn» dans les deux nuages). La plateforme constitue donc un espace bien différent de la base de données des collections destinée aux chercheurs ou aux gestionnaires, mais qui rend autonomes les contributeurs réguliers dans la consultation des différentes ressources documentaires botaniques, à commencer par celles de l'institution. La plateforme permet d'accompagner la consultation de l'information scientifique par les contributeurs et joue donc un rôle dans la médiation documentaire en facilitant la consultation des collections par des non-spécialistes. Il s'agit aussi d'un espace de médiation permettant de constituer un public à partir d'internautes non spécialistes (médiation culturelle) et de transmettre des connaissances documentaires par des cadrages implicites, des consignes explicites et des échanges (médiation des savoirs).
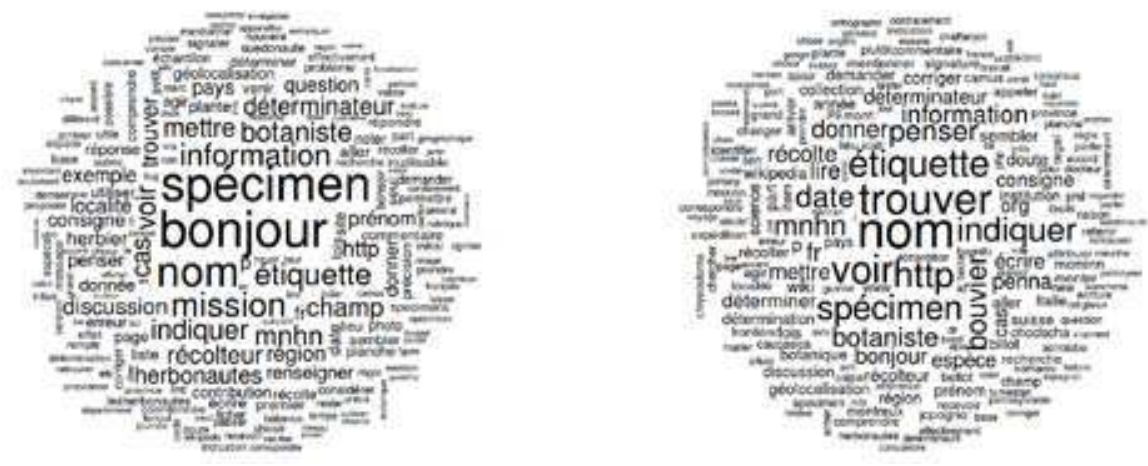

Figure 3 : (a)formes lexicales les plus fréquentes dans les forums de mission (nuage de gauche), et (b)formes lexicales les plus fréquentes dans les forums relatifs à chaque spécimen (nuage de droite). La taille des caractères et la distance au centre du nuage sont proportionnelles à la fréquence de la forme dans le corpus; le sens d'affichage du mot n'est pas signifiant. Paramètre de la réalisation du nuage avec Iramuteq (Ratinaud, 2005) : nombre maximum de formes $=200$. 
3.3. La découverte des collections médiatisées par les étiquettes: entre cadrage scientifique et subjectivité

L'interprétation des renseignements de l'étiquette par les internautes permet d'enregistrer dans la base de données une information scientifique: en plus de renseigner sur la composition de la collection, l'enregistrement du spécimen dans la base de données atteste de la présence de l'espèce à laquelle il appartient, à la date et dans la localité où le spécimen a été collecté. Cet apport de la contribution documentaire des internautes à l'exploitation d'informations d'intérêt scientifique se traduit par la possibilité de constituer automatiquement des cartes de répartition des spécimens transcrits dans une mission (voir figure 1). L'intérêt de la représentation cartographique issue des données de géolocalisation est particulièrement important quand l'itinéraire d'une expédition botanique ou d'un parcours d'herborisation peut être reconstitué grâce aux données des spécimens récoltés par une même personne. Ce cas montre comment les données obtenues grâce à l'étude collaborative d'une sélection de spécimens permettent de reconstituer les liens entre collections, personnes qui les ont produites et territoires qu'elles ont fréquentés. L'activité de transcription, qui conduit à retracer les parcours des botanistes, ouvre à l'acquisition de connaissances sur les acteurs de la constitution des collections et des savoirs botaniques.

L'examen de l'étiquette qui conduit à faire le lien entre la planche, son auteur, et le lieu de collecte, permet aussi de tisser un lien subjectif avec les collections, qui traduit une perception du spécimen non plus cadrée par les exigences scientifiques de la transcription, mais relative aux centres d'intérêt de l'internaute. C'est ce qui ressort des témoignages recueillis sur le sens donné à la participation. Les herbiers intéressent les contributeurs pour différentes raisons qui diffèrent principalement en fonction de leur rapport à la botanique. Si certains professionnels utilisent le site pour découvrir des flores qui les intéressent, chez d'autres contributeurs, c'est l'attachement à une région qui peut ainsi susciter l'intérêt pour les récoltes qui en proviennent: sauf dans les cas, rares mais notables, où un botaniste membre des "Herbonautes" se voit proposer un spécimen qu'il a lui-même récolté, ce n'est pas le fait que l'usager ait été témoin de la récolte qui va renforcer son intérêt, mais c'est le lien du spécimen à un territoire auquel il est lui-même attaché, et sur lequel le spécimen donne une information historique (l'état de la flore au moment de la collecte). Certains contributeurs insistent sur la connaissance du passé et des territoires à laquelle donne accès I'herbier: «J'aime (ré)apprendre la géographie française, d'une France qui souvent n'existe plus [...], lire qu'on parle des ruines du Château de Pierre-fonds parce que la récolte a été faite avant sa rénovation », témoigne une "herbonaute». Dans le cas évoqué, I’herbier est lui-même lié à d'autres patrimoines. Comprendre l'étiquette comme une «médiation" (Desjardins et Jacobi, 1992, p. 14) prend ici d'autant plus de sens qu'il ne s'agit pas seulement d'utiliser le document pour accéder à une information, mais pour construire une signification de l'objet en retrouvant le lien de l'herbier et «le monde des 
hommes qui l'ont produit, utilisé, codifié, embelli, voire au contraire saccagé ou détruit» (Davallon, 2006, p.123).

La plateforme permet donc de faire découvrir la valeur historique du spécimen et de mettre au service de la production de données documentaires cet intérêt suscité par l'herbier. Les motivations à participer et les sources de satisfaction qui les entretiennent sont à prendre en compte comme des conditions de l'efficacité du dispositif. La forte adhésion des répondants au projet se traduit dans les scores de satisfaction moyens élevés dans le questionnaire en ligne. Le registre le plus fréquemment exprimé dans les sources d'intérêt est celui de la "contribution » à un projet jugé «utile», d'intérêt général ou faisant progresser le travail scientifique. Cette motivation importante à participer à un projet utile et scientifique se retrouve dans les principales enquêtes réalisées sur les motivations des contributeurs de projets proposant des tâches à partir de documents scientifiques ou patrimoniaux par l'intermédiaire d'une plateforme ouverte à tous, dans le domaine des sciences de la nature (Raddick et al., 2010) ou des sciences humaines (Dunn et Hedges, 2014). Elle laisse envisager des traits communs aux collectifs mobilisés autour de projets participatifs visant à faciliter l'accessibilité, l'exploitation et la découverte des fonds numérisés pour différents publics et répartissant les fonctions de médiation entre le personnel de l'institution et ses publics.

La documentarisation participative des herbiers donne un exemple de convergence des objectifs et dispositifs associés à différentes traditions de métiers de médiation, documentaire, culturelle et scientifique. Le projet appelle des formes de discours, de documents, de collectifs et des normes relevant de ces différentes traditions. L'objectif documentaire de faciliter l'accès à l'information scientifique en enrichissant la base de données concourt à fédérer le collectif. La médiation scientifique à l'œuvre dans la plateforme se traduit par l'acquisition de connaissances relatives aux collections et à leur histoire; elle passe par les cadrages implicites de la participation ainsi que par l'animation scientifique collective qui reprend les codes de la vulgarisation scientifique, mais au service d'un projet qui renouvelle la position et le rôle du public par rapport à l'institution. L'acquisition des savoir-faire documentaires est indissociablement liée à l'opérativité sociale de la plateforme: des contributeurs de différents niveaux d'expertise sont mobilisés aux côtés des professionnels endossant le rôle de "chef de mission", figure originale de médiateur scientifique. Le dispositif opère un déplacement par rapport à la communication institutionnelle muséale en constituant un espace de médiation permettant aux profanes de s'approprier les techniques documentaires des collections scientifiques. La médiation des savoirs documentaires au sein de la communauté donne la possibilité de découvrir les herbiers comme des témoins de I'histoire de la botanique, des territoires et des personnes auxquels elle est liée; en retour ces découvertes nourrissent l'intérêt pour la participation et profitent à l'avancée du projet documentaire. 
Bibliographie

Casemajor-Loustau N. (2011). «La contribution triviale des amateurs sur le web: quelle efficacité documentaire?». In Études de communication, $n^{\circ} 36$, pp.39-52.

Chupin L. (2014). "Les dispositifs de transcription comme cadre de lecture. Le cas des herbiers collaboratifs ». In ZreikK., Azemard G., ChaudironS. et Darquié G. (dir.), Le Livre post-numérique, Actes du Colloque CIDE17, Paris, Europia Productions, pp.83-91.

Davallon J. (1992). «Le musée est-il vraiment un média?». In Publics et Musées, $n^{\circ} 2$, pp.99-123.

Davallon J. (1998). «Cultiver la science au musée? ». In SchieleB. et KosterE.(dir.), La Révolution de la muséologie des sciences, Lyon, Éditions Multimondes - Presses Universitaires de Lyon, pp.397-434.

Davallon J. (2004). «La communication en procès ». In Médiation et Information, $n^{\circ} 19$, pp.37-58.

Davallon J. (2006). Le Don du patrimoine, Paris, Hermès science publications.

Desjardins J. et JacobiD. (1992). "Les étiquettes dans les musées et les expositions scientifiques et techniques». In Publics et Musées, $n^{\circ} 1$, pp.13-32.
Després-Lonnet M. (2000). Contribution à la conception d'interfaces de consultation de bases de données iconographiques, thèse en sciences de l'information et de la communication, Université de Lille3, https://tel. archives-ouvertes.fr/tel-01155546v1, consulté en septembre 2015.

Després-Lonnet M. (2009). «L'écriture numérique du patrimoine, de l'inventaire à l'exposition: Les parcours de la base Joconde». In Culture \& Musées, n¹4, pp.19-38.

Després-Lonnet M. (2014). "L'écriture du patrimoine, de la documentation à la médiation». In Documentaliste-Sciences de I'Information, vol.51, pp.61-62.

Dunn S. et Hedges M. (2014). «How the Crowd Can Surprise Us: Humanities Crowdsourcing and the Creation of Knowledge». In Ridge M. (dir.), Crowdsourcing our Cultural Heritage, Surrey, Ashgate, pp.231-247.

E-ReColNat (2013), ReColNat [en ligne], http://ReColNat.org/, consulté en septembre 2015.

Fabrel. (2012). «Médiation documentaire et culturelle dans le musée». In Communication \& langages, $\mathrm{n}^{\circ} 173$, pp.83-99.

Haythornthwaite C. (2009). «Crowds and Communities: Light and Heavyweight Models of Peer Production ". In Proceedings of the Hawaii International Conference On System Sciences, Los Alamitos, États-Unis: IEEE Computer Society. 
Heaton L. et Proulx S. (2012). «La construction locale d'une base transnationale de données en botanique". In Revue d'anthropologie des connaissances, vol. 6, n¹, pp.141-162.

Jacobi D. (2007). «Communiquer par l'écrit dans les musées ». In SchieleB. et KosterE. (dir.), La révolution de la muséologie des sciences; des musées pour le XXIe siècle, Lyon, Éditions multimondes.

Liquète V., Fabrel. et Gardiès C. (2010). «Faut-il reconsidérer la médiation documentaire?». In Les Enjeux de l'information et de la communication, $n^{\circ} 2$, pp.43-57.

Muséum National d'Histoire Naturelle (2012). Les Herbonautes [en ligne], http://lesherbonautes.mnhn. $\mathrm{fr} /$, consulté en septembre 2015 .

Owens T. (2014). «Making crowdsourcing compatible with the missions and values of cultural heritage organisations ». In RidgeM. (dir), Crowdsourcing or cultural heritage, Surrey, Ashgate, pp.269-280.

PérezE. et Pignal M. (2013). «Numériser et promouvoir les collections d'histoire naturelle». In Bulletin des bibliothèques de France, n5, pp. 27-31.

RaddickM. J., Bracey G., GayP.L., Lintott C. J., Murray P., SchawinskiK., Szalay A.S. et Vandenberg J. (2010). «Galaxy Zoo: Exploring the Motivations of Citizen Science Volunteers ". In Astronomy Education Review, vol.9, n¹, http:// portico.org/stable?au=pgg3ztfdp8z, consulté en septembre 2015.

RatinaudP. (2005). Iramuteq [en ligne], http://www.iramuteq.org/, consulté en septembre 2015.

Ridge M. (2013). «From tagging to theorizing: deepening engagement with cultural heritage through crowdsourcing». In Curator: The Museum Journal, vol.56, n4 pp.435-450.

Salaün J.-M. (2007). «La redocumentarisation, un défi pour les sciences de l'information". In Études de communication, $\mathrm{n}^{\circ} 30,2007, \mathrm{pp} .13-23$.

SouchierE., Jeanneret Y. et Le Marec J. (dir.) (2003). Lire, écrire, récrire: objets, signes et pratiques des médias informatisés, Paris, Éditions de la Bibliothèque publique d'information.

Trant J. (2008). «Tagging, Folksonomy and Art Museums: Early Experiments and Ongoing Research ». In Journal of Digital Information, vol.10, $n^{\circ} 1$, http://journals. tdl.org/jodi/article/view/270/277, consulté en septembre 2015.

Welger-Barboza C. (2013). «Les catalogues de collection des musées en ligne, au carrefour des points de vue». In DufrêneB., Madjidl. et BruckmannD.(dir.), Numérisation du patrimoine. Quelles médiations? Quels accès? Quelles cultures?, Éditions Hermann, pp.191-214. 
Wiggins A. et Crowston K. (2011). «From Conservation to Crowdsourcing: A Typology of Citizen Science". In Proceedings of the $44^{\text {th }}$ Hawaii International Conference on System Science (HICSS-44), Kauai, Hawaii, 4-7janvier.

Zacklad M. et Chupin L. (2015). "Le crowdsourcing scientifique et patrimonial à la croisée de modèles de coordination et de coopération hétérogènes: le cas des herbiers numérisés». In Revue canadienne des sciences de l'information et de bibliothéconomie, n³9, pp.308-328.

Zacklad M. (2015). «Genres de dispositifs de médiation numérique et régimes de documentalité ». In Gagnon-Arguin L., MaurelD. et MasS.(dir.), Les genres de documents dans les organisations, analyse théorique et pratique, Montréal, PUQ, pp.145-184. 
